\title{
Integral pinching results for manifolds with boundary
}

\author{
Giovanni Catino And Cheikh BiRAhim Ndiaye
}

\begin{abstract}
We prove that some Riemannian manifolds with boundary satisfying an explicit integral pinching condition are spherical space-forms. More precisely, we show that three-dimensional Riemannian manifolds with totally geodesic boundary, positive scalar curvature and an explicit integral pinching between the $L^{2}$-norm of the scalar curvature and the $L^{2}$-norm of the Ricci tensor are spherical space-forms with totally geodesic boundary. Moreover, we also prove that four-dimensional Riemannian manifolds with umbilic boundary, positive Yamabe invariant and an explicit integral pinching between the total integral of the $(Q, T)$-curvature and the $L^{2}$-norm of the Weyl curvature are spherical space-forms with totally geodesic boundary. As a consequence, we show that a certain conformally invariant operator, which plays an important role in Conformal Geometry, is non-negative and has trivial kernel if the Yamabe invariant is positive and verifies a pinching condition together with the total integral of the $(Q, T)$-curvature. As an application of the latter spectral analysis, we show the existence of conformal metrics with constant $Q$-curvature, constant $T$-curvature, and zero mean curvature under the latter assumptions.
\end{abstract}

Mathematics Subject Classification (2010): 53C24 (primary); 53C20, 53C21, 53C25 (secondary).

\section{Introduction}

One of the most important questions about the relation between algebraic properties of the full curvature tensor and the topology of manifolds is under which conditions on its curvature tensor a Riemannian manifold is homeomorphic or diffeomorphic to a space of constant sectional curvature, namely a space form. A model example is the classical sphere theorem conjectured by Rauch [35], and which says that any closed, simply connected and $\frac{1}{4}$-pinched Riemannian manifold is diffeomorphic to the standard sphere. The topological version was proved by Berger [5] and Klingenberg [27]. Just recently the original conjecture has been settled by Brendle and Schoen [6], using a result of Bohm and Wilking [7].

On the other hand, many sphere like theorems appeared in the literature in the last 30 years in connection to the celebrated Ricci flow. Just to mention some of 
them which are related to our results, we start by recalling the pioneering work of R. Hamilton [22]. Using the Ricci flow, he proved the following theorem.

Theorem 1.1 (Hamilton). If (M,g) is a closed three-dimensional Riemannian manifold with positive Ricci curvature, then $M$ is diffeomorphic to a spherical space form, i.e. $M$ admits a metric with constant positive sectional curvature.

Later C. Margerin [30] proved an optimal curvature characterization of the smooth 4-sphere. We recall Margerin's theorem in a form where the optimality issue is not apparent, but enough for the link with our work. We define the weak pinching quantity

$$
W P_{g}=\frac{\left|W_{g}\right|_{g}^{2}+2\left|E_{g}\right|_{g}^{2}}{R_{g}^{2}},
$$

where $W_{g}$ denoting the Weyl tensor, $E_{g}$ the trace-free Ricci tensor and $|\cdot|_{g}$ the usual norm of a tensor with respect to the metric $g$. Here is the result.

Theorem 1.2 (Margerin). Let $(M, g)$ be a closed four-dimensional Riemannian manifold with positive scalar curvature. If the pinching condition $W P_{g}<\frac{1}{6}$ is satisfied, then $M$ is diffeomorphic to a spherical space form. Moreover, we get that the manifold $M$ is diffeomorphic to $S^{4}$ or $\mathbb{R} P^{4}$.

Much later, Chang, Gursky and Yang [15] proved a remarkable improvement of Margerin's theorem with assumptions which are in integral form, and conformally invariant too.

Theorem 1.3 (Chang-Gursky-Yang). Let $(M, g)$ be a closed four-dimensional Riemannian manifold with positive Yamabe invariant. If the curvatures satisfy

$$
\int_{M}\left(\left|W_{g}\right|_{g}^{2}+2\left|E_{g}\right|_{g}^{2}-\frac{1}{6} R_{g}^{2}\right) \mathrm{d} V_{g}<0,
$$

then $M$ is diffeomorphic to a spherical space form. Moreover, we get that the manifold $M$ is diffeomorphic to $S^{4}$ or $\mathbb{R} P^{4}$.

Notice that the integral pinching condition can be written in the following form (for the definition of $Q_{g}$, see below)

$$
\int_{M} Q_{g} \mathrm{~d} V_{g}>\frac{1}{8} \int_{M}\left|W_{g}\right|^{2} \mathrm{~d} V_{g} .
$$

Recently, the first author and Z. Djadli [9] proved an integral pinching theorem in dimension three.

Theorem 1.4 (Catino-Djadli). Let $(M, g)$ be a closed three-dimensional Riemannian manifold with positive scalar curvature. If

$$
\int_{M}\left|\operatorname{Ric}_{g}\right|_{g}^{2} \mathrm{~d} V \leq \frac{3}{8} \int_{M} R_{g}^{2} \mathrm{~d} V_{g},
$$

then $M$ is diffeomorphic to a spherical space form. 
A slightly weaker version of this result was also obtained by Y. Ge, C.-S. Lin and G. Wang [23].

On the other hand, the Ricci flow techniques have also been used to get sphere like theorems for manifolds with boundary. An example which is of interest to us is the following result of Shen [37].

Theorem 1.5 (Shen). If $(M, g)$ is a compact three-dimensional Riemannian manifold with totally geodesic boundary and positive Ricci curvature, then $M$ admits a metric with constant positive sectional curvatures in the interior and totally geodesic boundary.

Using the Ricci flow for manifolds with boundary defined by Shen [37], a very easy adaptation of the arguments of Margerin [30], immediately yields the following theorem:

Theorem 1.6. Let $(M, g)$ be a compact four-dimensional Riemannian manifold with totally geodesic boundary and positive scalar curvature. If the pinching condition $W P_{g}<\frac{1}{6}$ is satisfied, then $M$ admits a metric with constant positive sectional curvatures in the interior and totally geodesic boundary.

Our goal in this paper is to provide counterparts of the results of ChangGursky-Yang and Catino-Djadli for manifolds with boundary. The first result we will prove is the following:

Theorem 1.7. Let $(M, g)$ be a compact three-dimensional Riemannian manifold with totally geodesic boundary and positive scalar curvature. If

$$
\int_{M}\left|\operatorname{Ric}_{g}\right|_{g}^{2} \mathrm{~d} V_{g} \leq \frac{3}{8} \int_{M} R_{g}^{2} \mathrm{~d} V_{g},
$$

then $M$ admits a metric with constant positive sectional curvatures in the interior and totally geodesic boundary.

In order to state our second result on four manifolds with boundary, we need to recall some notions from Conformal Geometry. We start by recalling the Paneitz operator and its associated curvature invariant called $Q$-curvature. In 1983, Paneitz has discovered a conformally covariant differential operator on four dimensional compact smooth Riemannian manifolds with smooth boundary $(M, g)$ (see [33]). To this operator, Branson [4] has associated a natural curvature invariant called $Q$ curvature. They are defined in terms of Ricci tensor $\mathrm{Ric}_{g}$ and scalar curvature $R_{g}$ of the manifold $(M, g)$ as follows

$$
\begin{aligned}
P_{g}^{4} \varphi & =\Delta_{g}^{2} \varphi+\operatorname{div}_{g}\left(\left(\frac{2}{3} R_{g} g-2 \operatorname{Ric}_{g}\right) \mathrm{d} \varphi\right), \\
Q_{g} & =-\frac{1}{12}\left(\Delta_{g} R_{g}-R_{g}^{2}+3\left|\operatorname{Ric}_{g}\right|^{2}\right),
\end{aligned}
$$


where $\varphi$ is any smooth function on $M, \operatorname{div}_{g}$ is the divergence and $\mathrm{d}$ is the de Rham differential.

Similarly, Chang and Qing [13], have discovered a boundary operator $P_{g}^{3}$ defined on the boundary of compact four dimensional smooth Riemannian manifolds and a natural third-order curvature $T_{g}$ associated to $P_{g}^{3}$ as follows

$$
\begin{aligned}
P_{g}^{3} \varphi= & \frac{1}{2} \frac{\partial \Delta_{g} \varphi}{\partial n_{g}}+\Delta_{\hat{g}} \frac{\partial \varphi}{\partial n_{g}}-2 H_{g} \Delta_{\hat{g}} \varphi+\left(L_{g}\right)_{a b}\left(\nabla_{\hat{g}} \varphi\right)_{a}\left(\nabla_{\hat{g}} \varphi\right)_{b}+\nabla_{\hat{g}} H_{g} \cdot \nabla_{\hat{g}} \varphi \\
& +\left(F-\frac{R_{g}}{3}\right) \frac{\partial \varphi}{\partial n_{g}} . \\
T_{g}= & -\frac{1}{12} \frac{\partial R_{g}}{\partial n_{g}}+\frac{1}{2} R_{g} H_{g}-<G_{g}, L_{g}>+3 H_{g}^{3}-\frac{1}{3} \operatorname{Tr}\left(L^{3}\right)+\Delta_{\hat{g}} H_{g},
\end{aligned}
$$

where $\varphi$ is any smooth function on $M, \hat{g}$ is the metric induced by $g$ on $\partial M$, $L_{g}=\left(L_{g}\right)_{a b}=-\frac{1}{2} \frac{\partial g_{a b}}{\partial n_{g}}$ is the second fundamental form of $\partial M, H_{g}=\frac{1}{3} \operatorname{tr}\left(L_{g}\right)=$ $\frac{1}{3} g^{a b} L_{a b}$ ( $g^{a, b}$ are the entries of the inverse $g^{-1}$ of the metric $g$ ) is the mean curvature of $\partial M, R_{b c d}^{k}$ is the Riemann curvature tensor $F=R_{n a n}^{a}, R_{a b c d}=$ $g_{a k} R_{b c d}^{k}\left(g_{a, k}\right.$ are the entries of the metric $g$ ) and $\left\langle G_{g}, L_{g}\right\rangle=R_{a n b n}\left(L_{g}\right)_{a b}$, $\frac{\partial}{\partial n_{g}}$ is the inward normal derivative with respect to $g$. We recall that $(M, g)$ has umbilic boundary if $L_{g}=\lambda g$ for some constant $\lambda$. If $L_{g}=0$ we say that the boundary is totally geodesic.

A remarkable property of the couple of operators $\left(P_{g}^{4}, P_{g}^{3}\right)$ is that, as the couple Laplace-Beltrami operator and Neumann operator governs the transformation law of the Gauss curvature and the geodesic curvature on compact surfaces with boundary under conformal change of metric, $\left(P_{g}^{4}, P_{g}^{3}\right)$ does the same for $\left(Q_{g}, T_{g}\right)$ on compact four dimensional smooth Riemannian manifolds with boundary. In fact, after a conformal change of metric $g_{u}=e^{2 u} g$ we have that

$$
\left\{\begin{array} { l } 
{ P _ { g _ { u } } ^ { 4 } = e ^ { - 4 u } P _ { g } ^ { 4 } ; } \\
{ P _ { g _ { u } } ^ { 3 } = e ^ { - 3 u } P _ { g } ^ { 3 } ; }
\end{array} \quad \text { and } \quad \left\{\begin{array}{l}
P_{g}^{4} u+2 Q_{g}=2 Q_{g_{u}} e^{4 u} \\
P_{g}^{3} u+T_{g}=T_{g_{u}} e^{3 u}
\end{array} \quad \text { on } \quad \begin{array}{l}
M \\
\text { on }
\end{array}\right.\right.
$$

Another very important role played by the couple of curvatures $\left(Q_{g}, T_{g}\right)$ in Conformal Geometry is that they arise in the well-known Gauss-Bonnet-Chern formula. More precisely

$$
\int_{M}\left(Q_{g}+\frac{\left|W_{g}\right|^{2}}{8}\right) \mathrm{d} V_{g}+\oint_{\partial M}(T+Z) \mathrm{d} S_{g}=4 \pi^{2} \chi(M)
$$

where $W_{g}$ and $Z \mathrm{~d} S_{g}$ (for the definition of $Z$ see [13]) are pointwise conformally invariant. Moreover, it turns out that $Z$ vanishes when the boundary is totally geodesic. Setting

$$
\kappa_{P_{g}^{4}}=\int_{M} Q_{g} \mathrm{~d} V_{g}, \quad \kappa_{P_{g}^{3}}=\oint_{\partial M} T_{g} \mathrm{~d} S_{g}
$$


from (1.2), thanks to the fact that $W_{g} \mathrm{~d} V_{g}$ and $Z \mathrm{~d} S_{g}$ are pointwise conformally invariant, we have that $\kappa_{P_{g}^{4}}+\kappa_{P_{g}^{3}}$ is conformally invariant, and will be denoted by

$$
\kappa_{\left(P^{4}, P^{3}\right)}=\kappa_{P_{g}^{4}}+\kappa_{P_{g}^{3}} .
$$

In addition to the conformally invariant quantity $\kappa_{\left(P^{4}, P^{3}\right)}$ of a compact four-dimensional Riemannian manifold with boundary, there exists also the Yamabe invariant of the conformal class $[g]=\left\{\tilde{g}=e^{2 u} g, \quad u \in C^{\infty}(M)\right\}$ defined by the

$$
Y(M, \partial M,[g])=\inf _{\tilde{g} \in[g], \mathrm{vol}_{\tilde{g}}=1} \int_{M} R_{\tilde{g}} \mathrm{~d} V_{\tilde{g}}+\oint_{\partial M} H_{\tilde{g}} \mathrm{~d} S_{\tilde{g}} .
$$

We recall that this invariant is defined for every compact Riemannian manifold with boundary of dimension greater or equal to 3 .

Now we are ready to state our result on four manifolds with boundary.

Theorem 1.8. Let $(M, g)$ be a compact four-dimensional Riemannian manifold with umbilic boundary. If $Y(M, \partial M,[g])>0$ and if $\kappa_{\left(P^{4}, P^{3}\right)}>\frac{1}{8} \int_{M}\left|W_{g}\right|^{2} \mathrm{~d} V_{g}$, then $M$ admits a metric with constant positive sectional curvatures in the interior and totally geodesic boundary.

The couple $\left(P_{g}^{4}, P_{g}^{3}\right)$ gives rise to an operator defined on $H_{\frac{\partial}{\partial n}}=\left\{u \in H^{2}(M)\right.$ : $\left.\frac{\partial u}{\partial n_{g}}=0\right\}$ whose spectral property is very important for uniformization problems on four manifolds with boundary. The latter operator that we denote by $P_{g}^{4,3}$ is defined as follows

$$
\begin{aligned}
\left\langle P_{g}^{4,3} u, v\right\rangle_{L^{2}(M)}= & \int_{M}\left(\Delta_{g} u \Delta_{g} v+\frac{2}{3} R_{g} \nabla_{g} u \nabla_{g} v\right) \mathrm{d} V_{g} \\
& -2 \int_{M} \operatorname{Ric}_{g}\left(\nabla_{g} u, \nabla_{g} v\right) \mathrm{d} V_{g}-2 \oint_{\partial M} L_{g}\left(\nabla_{\hat{g}} u, \nabla_{\hat{g}} v\right) \mathrm{d} S_{g},
\end{aligned}
$$

for every $u, v \in H_{\frac{\partial}{\partial n}}$.

As a byproduct of our analysis, we obtain the following spectral property for $P_{g}^{4,3}$.

Theorem 1.9. Let $(M, g)$ be a compact four-dimensional Riemannian manifold with umbilic boundary. Assuming $Y(M, \partial M,[g])>0$ and $\kappa_{\left(P^{4}, P^{3}\right)}+\frac{1}{6} Y(M, \partial M$, $[g])^{2}>0$, then $P_{g}^{4,3}$ is non-negative and $\operatorname{ker} P_{g}^{4,3} \simeq \mathbb{R}$.

A direct consequence of Theorem 1.9 is the existence of constant $Q$-curvature and constant $T$-curvature conformal metrics on four-manifolds which verify the assumptions of Theorem 1.9 
Corollary 1.10. Let $(M, g)$ be a compact four-dimensional Riemannian manifold with umbilic boundary. Assuming $Y(M, \partial M,[g])>0$ and $\kappa_{\left(P^{4}, P^{3}\right)}+\frac{1}{6} Y(M$, $\partial M,[g])^{2}>0$, then $M$ carries a metric conformal to $g$ with constant $Q$-curvature, constant $T$-curvature and zero mean curvature.

Proofs of Theorem 1.7 and Theorem 1.8 rely on the solution of some boundary value problems for fully nonlinear equations. Following [26] we will use the continuity method proving a priori estimates on the solutions to our equations. As a consequence of our work in dimension four, analising the spectral property of a certain operator, we will show that this operator is non-negative and with trivial kernel (Theorem 1.9). As a byproduct, we will prove then existence of conformal metrics with constant $Q$-curvature, constant $T$-curvature and zero mean curvature under certain conformally invariant assumptions (Corollary 1.10).

The plan of the paper is the following: in Section 2 we will introduce some notations, set up the boundary value problem; in Section 3 and 4 we will prove Theorem 1.7 and Theorem 1.8 on three and four manifolds respectively; finally Section 5 will be devote to the proof of Theorem 1.9 and Corollary 1.10.

\section{Preliminaries and notation}

In this section, we give some notation and preliminaries like the notion of $k$-th symmetric elementary functions and some of their properties, the notion of $\sigma_{k}$ curvature of a Riemannian manifold, and some Moser-Trudinger type inequalities. For this end, let $(M, g)$ be a compact, smooth, $n$-dimensional Riemannian manifold with boundary. We will denote by $v_{g}$ the inner normal vector field with respect to the metric $g$ and by $\partial_{\nu}=\frac{\partial}{\partial n_{g}}$ the inward normal derivative. Moreover $L_{g}$ and $H_{g}$ will be the second fundamental form

$$
L_{g, a b}=-\frac{1}{2} \frac{\partial g_{a b}}{\partial n_{g}},
$$

and the mean curvature normalized, i.e.

$$
H_{g}=\frac{1}{n-1} g^{a b} L_{g, a b} .
$$

Given a section $A$ of the bundle of symmetric 2-tensors, we can use the metric to raise an index and view $A$ as a tensor of type $(1,1)$, or equivalently as a section of $\operatorname{End}(T M)$. This allows us to define $\sigma_{k}\left(g^{-1} A\right)$ the $k$-th elementary function of the eigenvalues of $g^{-1} A$. More precisely we define:

Definition 2.1. Let $\left(\lambda_{1}, \cdots, \lambda_{n}\right) \in \mathbb{R}^{n}$. We view the $k$-th elementary symmetric function as a function on $\mathbb{R}^{n}$ :

$$
\sigma_{k}\left(\lambda_{1}, \cdots, \lambda_{n}\right)=\sum_{1 \leq i_{1}<\cdots<i_{k} \leq n} \lambda_{i_{1}} \cdots \lambda_{i_{k}}
$$


and we define

$$
\Gamma_{k}^{+}=\bigcap_{1 \leq j \leq k}\left\{\sigma_{j}\left(\lambda_{1}, \cdots, \lambda_{n}\right)>0\right\} \subset \mathbb{R}^{n} .
$$

For a symmetric linear transformation $A: V \rightarrow V$, where $V$ is an $n$-dimensional inner product space, the notation $A \in \Gamma_{k}^{+}$will mean that the eigenvalues of $A$ lie in the corresponding set. We note that this notation also makes sense for a symmetric 2-tensor on a Riemannian manifold. If $A \in \Gamma_{k}^{+}$, let $\sigma_{k}^{1 / k}(A)=\left\{\sigma_{k}(A)\right\}^{1 / k}$.

Definition 2.2. Let $A: V \rightarrow V$, where $V$ is an $n$-dimensional inner product space. The $(k-1)$-th Newton transformation associated with $A$ is

$$
T_{(k-1)}(A)=\sum_{j=0}^{k-1}(-1)^{k-1-j} \sigma_{j}(A) A^{k-1-j} .
$$

Also, for $t \in \mathbb{R}$ we define the linear transformation

$$
L^{t}(A)=T_{(k-1)}(A)+\frac{1-t}{n-2} \sigma_{1}\left(T_{(k-1)}(A)\right) \cdot I .
$$

We have the following list of properties (the proofs can be found in [8])

\section{Lemma 2.3.}

(i) $\Gamma_{k}^{+}$is an open convex cone with vertex at the origin, and we have the following sequence of inclusions

$$
\Gamma_{n}^{+} \subset \Gamma_{n-1}^{+} \subset \cdots \subset \Gamma_{1}^{+}
$$

(ii) If $A \in \Gamma_{k}^{+}$, then $T_{k-1}(A)$ is positive definite. Hence for all $t \leq 1, L^{t}(A)$ is positive definite.

(iii) We have the identities

$$
\begin{gathered}
T_{k-1}(A)^{i j} A_{i j}=k \sigma_{k}(A), \\
T_{k-1}(A)^{l l}=(n-k+1) \sigma_{k-1}(A) .
\end{gathered}
$$

(iv) If $A \in \Gamma_{k}^{+}$, then

$$
\sigma_{k-1}(A) \geq \frac{k}{n-k+1}\left(\begin{array}{l}
n \\
k
\end{array}\right)^{\frac{1}{k}} \sigma_{k}(A)^{\frac{(k-1)}{k}} .
$$

(v) If $A$ and $B$ are symmetric linear transformations, $A, B \in \Gamma_{k}^{+}$, then $\forall \rho \in$ $[0,1], \rho A+(1-\rho) B \in \Gamma_{k}^{+}$, and

$$
\sigma_{k}^{\frac{1}{k}}(\rho A+(1-\rho) B) \geq \rho \sigma_{k}^{\frac{1}{k}}(A)+(1-\rho) \sigma_{k}^{\frac{1}{k}}(B) .
$$

In particular this gives the concavity of the function $\sigma_{k}^{\frac{1}{k}}$ in the cone $\Gamma_{k}^{+}$. 
Next we give a lemma about the variation of the $\sigma_{k}$ functional.

Lemma 2.4. If $A: \mathbb{R} \rightarrow \operatorname{Hom}(V, V)$, then

$$
\frac{\mathrm{d}}{\mathrm{d} s} \sigma_{k}(A)(s)=\sum_{i, j} T_{(k-1)}(A)_{i j}(s) \frac{\mathrm{d}}{\mathrm{d} s}(A)_{i j}(s),
$$

i.e., the $(k-1)$-th Newton transformation is what arises when we differentiate $\sigma_{k}$.

We choose the tensor (here $t$ is a real number)

$$
A_{g}^{t}=\frac{1}{n-2}\left(\operatorname{Ric}_{g}-\frac{t}{2(n-1)} R_{g} g\right),
$$

where $\operatorname{Ric}_{g}$ and $R_{g}$ denote the Ricci and the scalar curvature of $g$ respectively. Note that for $t=1, A_{g}^{1}$ is the classical Schouten tensor, namely $A_{g}^{1}=A_{g}:=$ $\frac{1}{n-2}\left(\operatorname{Ric}_{g}-\frac{1}{2(n-1)} R_{g} g\right)$, see [1]. Hence, with our notations, $\sigma_{k}\left(g^{-1} A_{g}^{t}\right)$ denotes the $k$-th elementary symmetric function of the eigenvalues of $g^{-1} A_{g}^{t}$.

Now, we give a lemma which shows that metrics $g_{1}$, such that $A_{g_{1}}^{t}$ belong to the positive cone of order 2 , verify also additional pointwise algebraic inequalities. More precisely, we have:

Lemma 2.5. If for some metric $g_{1}$ on $M$ we have $A_{g_{1}}^{t} \in \Gamma_{2}^{+}$, then

$$
\begin{aligned}
-A_{g_{1}}^{t}+\sigma_{1}\left(g_{1}^{-1} A_{g_{1}}^{t}\right) g_{1} & >0, \\
A_{g_{1}}^{t}+\frac{n-2}{n} \sigma_{1}\left(g_{1}^{-1} A_{g_{1}}^{t}\right) g_{1} & >0 .
\end{aligned}
$$

We will be concerned with the following equation for a conformal metric $\tilde{g}=$ $e^{-2 u} g$ :

$$
\begin{cases}\sigma_{k}^{1 / k}\left(g^{-1} A_{u}^{t}\right)=f e^{2 u} & \text { in } \quad M, \\ \partial_{\nu} u=0 & \text { on } \quad \partial M .\end{cases}
$$

Where $f$ is a positive function on $M$. Let $\sigma_{1}\left(g^{-1} A_{g}^{1}\right)$ be the trace of $A_{g}^{1}$ with respect to the metric $g$. We have the following formula for the transformation of $A_{g}^{t}$ under this conformal change of metric:

$$
A_{\tilde{g}}^{t}=A_{g}^{t}+\nabla_{g}^{2} u+\frac{1-t}{n-2}\left(\Delta_{g} u\right) g+\mathrm{d} u \otimes \mathrm{d} u-\frac{2-t}{2}\left|\nabla_{g} u\right|_{g}^{2} g .
$$

Since

$$
A_{g}^{t}=A_{g}^{1}+\frac{1-t}{n-2} \sigma_{1}\left(g^{-1} A_{g}^{1}\right) g
$$


this formula follows easily from the standard formula for the transformation of the Schouten tensor [36]:

$$
A_{\tilde{g}}^{1}=A_{g}^{1}+\nabla_{g}^{2} u+\mathrm{d} u \otimes \mathrm{d} u-\frac{1}{2}\left|\nabla_{g} u\right|_{g}^{2} g .
$$

Using this formula we may write (2.1) with respect to the background metric $g$

$$
\sigma_{k}\left(g^{-1}\left(A_{g}^{t}+\nabla_{g}^{2} u+\frac{1-t}{n-2}\left(\Delta_{g} u\right) g+\mathrm{d} u \otimes \mathrm{d} u-\frac{2-t}{2}\left|\nabla_{g} u\right|_{g}^{2} g\right)\right)^{1 / k}=f(x) e^{2 u} .
$$

Now, we discuss the ellipticity properties of equation (2.1).

Proposition 2.6 (Ellipticity property). Let $u \in C^{2}(M)$ be a solution of equation (2.1) for some $t \leq 1$ and let $\tilde{g}=e^{-2 u}$ g. Assume that $A_{\tilde{g}}^{t} \in \Gamma_{k}^{+}$. Then the linearized operator at $u, \mathcal{L}^{t}: C^{2, \alpha}(M) \cap\left\{\partial_{\nu} u=0\right.$ on $\left.\partial M\right\} \rightarrow C^{\alpha}(M)$, is elliptic and invertible $(0<\alpha<1)$.

Proof. Define the operator

$$
F_{t}\left[u, \nabla_{g} u, \nabla_{g}^{2} u\right]=\sigma_{k}\left(g^{-1} A_{\tilde{g}}^{t}\right)-f(x)^{k} e^{2 k u},
$$

so that solutions of the equation (2.1) are exactly the zeroes of $F_{t}$. Define the function $u_{s}=u+s \varphi$, then the linearization at $u$ of the operator $F_{t}$ is defined by

$$
\begin{aligned}
\mathcal{L}^{t}(\varphi) & =\left.\frac{\mathrm{d}}{\mathrm{d} s} F_{t}\left[u_{s}, \nabla_{g} u_{s}, \nabla_{g}^{2} u_{s}\right]\right|_{s=0} \\
& =\left.\frac{\mathrm{d}}{\mathrm{d} s}\left(\sigma_{k}\left(g^{-1} A_{\tilde{g}}^{t}\right)\right)\right|_{s=0}-\left.\frac{\mathrm{d}}{\mathrm{d} s}\left(f(x)^{k} e^{2 k u_{s}}\right)\right|_{s=0} .
\end{aligned}
$$

From Lemma 2.4 we have

$$
\left.\frac{\mathrm{d}}{\mathrm{d} s}\left(\sigma_{k}\left(g^{-1} A_{\tilde{g}}^{t}\right)\right)\right|_{s=0}=\left.T_{k-1}\left(g^{-1} A_{\tilde{g}}^{t}\right)_{i j} \frac{\mathrm{d}}{\mathrm{d} s}\left(\left(A_{\tilde{g}}^{t}\right)_{i j}\right)\right|_{s=0} .
$$

We compute

$$
\left.\frac{\mathrm{d}}{\mathrm{d} s}\left(\left(A_{\tilde{g}}^{t}\right)_{i j}\right)\right|_{s=0}=\left(\nabla_{g}^{2} \varphi\right)_{i j}+\frac{1-t}{n-2}\left(\Delta_{g} \varphi\right) g_{i j}-(2-t) \nabla_{g} u \cdot \nabla_{g} \varphi g_{i j}+2 d u \otimes d \varphi .
$$

Easily we have also

$$
\left.\frac{\mathrm{d}}{\mathrm{d} s}\left(f(x)^{k} e^{2 k u_{s}}\right)\right)\left.\right|_{s=0}=2 k f(x)^{k} e^{2 k u} \varphi .
$$

Putting all together, we conclude

$$
\mathcal{L}^{t}(\varphi)=T_{k-1}\left(g^{-1} A_{\tilde{g}}^{t}\right)_{i j}\left(\left(\nabla_{g}^{2} \varphi\right)_{i j}+\frac{1-t}{n-2}\left(\Delta_{g} \varphi\right) g_{i j}\right)-2 k f(x)^{k} e^{2 k u} \varphi+\cdots
$$


where the last terms denote additional ones witch are linear in $\nabla_{g} \varphi$. The first term of the linearization is exactly the one defined in Definition 2.2, i.e.

$$
L^{t}\left(A_{\tilde{g}}^{t}\right)_{i j}=T_{k-1}\left(A_{\tilde{g}}^{t}\right)_{i j}+\frac{1-t}{n-2} T_{k-1}\left(A_{\tilde{g}}^{t}\right)_{p p} \delta_{i j}
$$

So finally, we have

$$
\mathcal{L}^{t}(\varphi)=L^{t}\left(A_{\tilde{g}}^{t}\right)_{i j}\left(\nabla_{g}^{2} \varphi\right)_{i j}-2 k f(x)^{k} e^{2 k u} \varphi+\cdots
$$

Since $A_{\tilde{g}}^{t} \in \Gamma_{k}^{+}$, by Lemma 2.3, we have that the tensor $L^{t}\left(A_{\tilde{g}}^{t}\right)$ is positive definite. So, the linearized operator at any solution $u$ must be elliptic. Note also that, by the previous formula, the operator is of the form

$$
\mathcal{L}^{t}(\varphi)=E(\varphi)-c(x) \varphi,
$$

where $E(\varphi)$ is a second order linear elliptic operator and $c(x)$ is a strictly positive function on $M$, since $c(x)=2 k f(x)^{k} e^{2 k u}$ and $f(x)>0$. This allows us to invert this operator between the Hölder spaces $C^{2, \alpha}(M) \cap\left\{\partial_{\nu} u=0\right.$ on $\left.\partial M\right\}$ and $C^{\alpha}(M)$ (see for instance [24]).

Next, we recall some Moser-Trudinger type inequalities which will be used to prove Corollary 1.10 .

Proposition 2.7. Assume $(M, g)$ is a compact four-dimensional Riemannian manifold with boundary such that $P_{g}^{4,3}$ is a non-negative operator with $\operatorname{Ker} P_{g}^{4,3} \simeq \mathbb{R}$. Then we have that for all $\alpha<16 \pi^{2}$ there exists a constant $C=C(M, g, \alpha)$ such that

$$
\int_{M} e^{\frac{\alpha(u-\bar{u})^{2}}{\left\langle P_{g}^{4,3} u, u\right\rangle_{L^{2}(M)}}} \mathrm{d} V_{g} \leq C,
$$

for all $u \in H_{\frac{\partial}{\partial n}}$, and hence

$$
\log \int_{M} e^{4(u-\bar{u})} \leq C+\frac{4}{\alpha}\left\langle P_{g}^{4,3} u, u\right\rangle_{L^{2}(M)} \quad \forall u \in H_{\frac{\partial}{\partial n}},
$$

where $\bar{u}=\frac{1}{\operatorname{Vol}_{g}(M)} \int_{M} u \mathrm{~d} V_{g}$, and $\operatorname{Vol}_{g}(M)=\int_{M} \mathrm{~d} V_{g}$.

The latter proposition can be found in [31] together with its proof. The second inequality that we are going to state is a trace analogue of the previous one. Its proof can be found [32].

Proposition 2.8. Assume $P_{g}^{4,3}$ is a non-negative operator with $\operatorname{Ker} P_{g}^{4,3} \simeq \mathbb{R}$. Then we have that for all $\alpha<12 \pi^{2}$ there exists a constant $C=C(M, g, \alpha)$ such that

$$
\oint_{\partial M} e^{\frac{\alpha\left(u-\bar{u}_{\partial M}\right)^{2}}{\left\langle P_{g}^{4,3} u,\left.u\right|_{L^{2}(M, g)}\right.}} \mathrm{d} S_{g} \leq C,
$$


for all $u \in H_{\frac{\partial}{\partial n}}$, and hence

$$
\log \oint_{\partial M} e^{3\left(u-\bar{u}_{\partial M}\right)} \mathrm{d} S_{g} \leq C+\frac{9}{4 \alpha}\left\langle P_{g}^{4,3} u, u\right\rangle_{L^{2}(M, g)} \quad \forall u \in H_{\frac{\partial}{\partial n}} .
$$

where $\bar{u}_{\partial M}=\frac{1}{\operatorname{Vol}_{g}(\partial M)} \oint_{\partial M} u \mathrm{~d} S_{g}$, and $\operatorname{Vol}_{g}(\partial M)=\oint_{\partial M} \mathrm{~d} S_{g}$.

Now, we give a lemma (whose proof can be found in [31]) which will be used together with the above Moser-Trudinger type inequalities in order to prove Corollary 1.10. It says that under the assumptions $\operatorname{Ker} P_{g}^{4,3} \simeq \mathbb{R}$ and $P_{g}^{4,3}$ non-negative, the map

$$
u \in H_{\frac{\partial}{\partial n}} \longrightarrow\|u\|_{P_{g}^{4,3}}=\left\langle P_{g}^{4,3} u,\left.u\right|_{L^{2}(M)} ^{\frac{1}{2}}\right.
$$

induces an equivalent norm to the standard norm of $H^{2}(M)$ on $\left\{u \in H_{\frac{\partial}{\partial n}} \bar{u}=0\right\}$. More precisely we have the following:

Lemma 2.9. Suppose $\operatorname{Ker} P_{g}^{4,3} \simeq \mathbb{R}$ and $P_{g}^{4,3}$ non-negative then we have that $\|\cdot\|_{P_{g}^{4,3}}$ is an equivalent norm to $\|\cdot\|_{H^{2}}$ on $\left\{u \in H_{\frac{\partial}{\partial n}} \bar{u}=0\right\}$. rems.

Now we give a technical lemma which will be used to prove the above theo-

Lemma 2.10. Let $(M, g)$ be a compact $n$-dimensional Riemannian manifold with totally geodesic boundary. Assuming $u \in C^{2}(M)$ with $\frac{\partial u}{\partial n_{g}}=0$, then

$$
\frac{\partial\left|\nabla_{g} u\right|_{g}^{2}}{\partial n_{g}}=0
$$

and

$$
A_{g}\left(\nu, \nabla_{g} u\right)=0 .
$$

Proof. First of all, using the fact that $\frac{\partial u}{\partial n_{g}}=0$, we derive

$$
\left|\nabla_{g} u\right|^{2}=g^{a b} \partial_{a} u \partial_{b} u .
$$

Thus we infer

$$
\frac{\partial\left(\left|\nabla_{g} u\right|^{2}\right)}{\partial n_{g}}=\frac{\partial g^{a b}}{\partial n_{g}} \partial_{a} u \partial_{b} u+2 g^{a b} \frac{\partial\left(\partial_{a} u\right)}{\partial n_{g}} \partial_{b} u
$$

Next, using the fact that $L_{g}=0$, one has $\frac{\partial g^{a b}}{\partial n_{g}}=0$. Moreover from the trivial identity $\frac{\partial\left(\partial_{a} u\right)}{\partial n_{g}}=\partial_{a}\left(\frac{\partial u}{\partial n_{g}}\right)$, we infer

$$
\frac{\partial\left(\partial_{a} u\right)}{\partial n_{g}}=0
$$


Thus, we obtain

$$
\frac{\partial\left(\left|\nabla_{g} u\right|^{2}\right)}{\partial n_{g}}=0
$$

This prove the first point. For the second one, we have

$$
A_{g}\left(\nu, \nabla_{g} u\right)=\frac{1}{n-2}\left(\operatorname{Ric}_{g}\left(\nu, \nabla_{g} u\right)-\frac{1}{2(n-1)} R_{g} \frac{\partial u}{\partial n_{g}}\right) \text {. }
$$

Thus, we get

$$
A_{g}\left(\nu, \nabla_{g} u\right)=\frac{1}{n-2} \operatorname{Ric}_{v, a} \partial_{a} u .
$$

Now using the Codazzi-Mainardi equation, we get

$$
\operatorname{Ric}_{v, a}=\nabla_{b} L_{g, a b}-\nabla_{a} H_{g}=0 .
$$

So, we obtain.

$$
A_{g}\left(\nu, \nabla_{g} u\right)=0 .
$$

This completes the proof of the lemma.

\section{Three manifolds with boundary}

In this section, we present the proof of Theorem 1.7. We will prove a more general theorem so that Theorem 1.7 will be a direct corollary. In fact, we have:

Theorem 3.1. Let $(M, g)$ be a compact three-dimensional Riemannian manifold with totally geodesic boundary and positive scalar curvature. There exists a positive constant $C=C\left(\operatorname{diam}(M, g),\left\|\nabla^{2} R m\right\|\right)$ such that if

$$
\int_{M} \sigma_{2}\left(g^{-1} A_{g}^{1}\right) \mathrm{d} V_{g}+C\left(\frac{7}{10}-t_{0}\right) Y(M,[g])^{2}>0,
$$

for some $t_{0} \leq 2 / 3$, then there exists a conformal metric $\tilde{g}=e^{-2 u} g$ with $R_{\tilde{g}}>0$, $\sigma_{2}\left(g^{-1} A_{\tilde{g}}^{t_{0}}\right)>0$ pointwise and totally geodesic boundary. Moreover, we have the inequalities

$$
\left(3 t_{0}-2\right) R_{\tilde{g}} \tilde{g}<6 \operatorname{Ric}_{\tilde{g}}<3\left(2-t_{0}\right) R_{\tilde{g}} \tilde{g}
$$

Throughout the sequel, $(M, g)$ will be a compact 3-dimensional Riemannian manifold with totally geodesic boundary and with positive scalar curvature. Since $M$ is compact and $R_{g}>0$, there exists $t_{0}>\delta>-\infty$ such that $A_{g}^{\delta}$ is positive definite (i.e. $\operatorname{Ric}_{g}-\frac{\delta}{4} R_{g} g>0$ on $M$ ). Note that $\delta$ only depends on $\left\|R m_{g}\right\|$. 
For $t \in\left[\delta, t_{0}\right]$, consider the path of equations (in the sequel we use the notation $A_{u_{t}}^{t}:=A_{g_{t}}^{t}$ for $g_{t}$ given by $g_{t}=e^{-2 u_{t}} g$ )

$$
\begin{cases}\sigma_{2}^{1 / 2}\left(g^{-1} A_{u_{t}}^{t}\right)=f e^{2 u_{t}} & \text { in } \quad M, \\ \partial_{\nu} u_{t}=0 & \text { on } \partial M\end{cases}
$$

where $f=\sigma_{2}^{1 / 2}\left(g^{-1} A_{g}^{\delta}\right)>0$. Note that $u \equiv 0$ is a solution for $t=\delta$.

We use the continuity method. Define

$$
\mathcal{S}=\left\{t \in\left[\delta, t_{0}\right] \mid \exists \text { a solution } u_{t} \in C^{2, \alpha}(M) \text { of (3.2) with } A_{u_{t}}^{t} \in \Gamma_{2}^{+}\right\}
$$

Clearly, with our choice of $f, u \equiv 0$ is a solution for $t=\delta$. Since $A_{g}^{\delta}$ is positive definite, then $\delta \in \mathcal{S}$. Hence $\mathcal{S} \neq \emptyset$. Let $t \in \mathcal{S}$, and $u_{t}$ be a solution. By Proposition 2.6, the linearized operator at $u_{t}, \mathcal{L}^{t}: C^{2, \alpha}(M) \cap\left\{\partial_{\nu} u=0\right.$ on $\left.\partial M\right\} \rightarrow C^{\alpha}(M)$, is invertible. The implicit function theorem tells us that $\mathcal{S}$ is open. To prove that $\mathcal{S}$ is close we need to establish a priori $C^{2, \alpha}$ estimates for solutions of the equation (3.2). To do this, we start by proving an upper bound estimate for solutions of (3.2).

Proposition 3.2 (Upper bound). Let $u_{t} \in C^{2}(M)$ be a solution of (3.2) for some $t \in\left[\delta, t_{0}\right]$. If $g_{t}=e^{-2 u_{t}} g \in \Gamma_{2}^{+}$, then $u_{t} \leq \bar{\delta}$, where $\bar{\delta}$ depends only on $\left\|R m_{g}\right\|$.

Proof. From Lemma 2.3 (iv), we have $\sqrt{3} \sigma_{2}^{1 / 2} \leq \sigma_{1}$, so for all $p \in M$

$$
\sqrt{3} f e^{2 u_{t}} \leq \sigma_{1}\left(g^{-1} A_{u_{t}}^{t}\right)
$$

Let $p \in M$ be a maximum of $u_{t}$. Since the gradient terms vanish at $p$ (this is true also if $p \in \partial M$, since $\partial_{\nu} u_{t}=0$ on $\left.\partial M\right)$ we have $\left(\Delta u_{t}\right)(p) \leq 0$. Then, using (2.2), we have

$$
\begin{aligned}
\sqrt{3} f(p) e^{2 u_{t}(p)} & \leq \sigma_{1}\left(g^{-1} A_{u_{t}}^{t}\right)(p) \\
& =\sigma_{1}\left(g^{-1} A_{g}^{t}\right)(p)+(4-3 t)\left(\Delta u_{t}\right)(p) \\
& \leq \sigma_{1}\left(g^{-1} A_{g}^{t}\right)(p) \\
& \leq \sigma_{1}\left(g^{-1} A_{g}^{\delta}\right)(p) .
\end{aligned}
$$

Since $M$ is compact, we have $u_{t} \leq \bar{\delta}$, for some $\bar{\delta}$ depending only on $\left\|R m_{g}\right\|$.

Next, we are going to show that solutions of (3.2) which verify upper-bound estimates enjoy also gradient ones:

Proposition 3.3 (Gradient estimate). Let $u_{t} \in C^{3}(M)$ be a solution of (3.2) for some $\delta \leq t \leq t_{0}$. Assume that $u_{t} \leq \bar{\delta}$. Then $\left\|\nabla_{g} u\right\|_{g, \infty}<C_{1}$, where $C_{1}$ depends only on $\left\|\nabla R m_{g}\right\|$ and $\bar{\delta}$. 
Proof. Let $H:=\left|\nabla_{g} u\right|_{g}^{2}$. If the maximum of $H$ is in the interior, then $\nabla_{g} H=$ 0 and $\nabla_{g}^{2} H$ is negative semi-definite. If the maximum of $H$ is at the boundary, then by Lemma 2.10, $\frac{\partial H}{\partial n_{g}}=0$. Thus, we also have that $\nabla_{g} H=0$ and $\nabla_{g}^{2} H$ is negative semi-definite. Interior gradient estimates for equation (3.2) were proved in [26, Proposition 4.1]. We remark that the same proof works for boundary gradient estimates. The reason is that, as we showed, at the maximal point once we have $\nabla_{g} H=0$ and $\nabla_{g}^{2} H$ is negative semi-definite, then the rest of computations in [26] is the same regardless of the point being in the interior or on the boundary.

As we proved before, there exist two constants $\bar{\delta}$ and $C_{1}$ depending only on $\left\|\nabla R m_{g}\right\|$ such that all solutions of (3.2) for some $\delta \leq t \leq t_{0}$, satisfying $u_{t} \leq \bar{\delta}$ satisfy $\left\|\nabla_{g} u\right\|_{\infty}<C_{1}$. Consider now the following quantity:

$$
I(M, \partial M, g):=\inf _{g^{\prime}=e^{-2 \varphi} g,\left|\nabla_{g} \varphi\right| \leq C_{1}, H_{g^{\prime}}=0}\left(\int_{M} R_{g^{\prime}}^{2} e^{-\varphi} \mathrm{d} V_{g^{\prime}}\right) .
$$

We let, for $g^{\prime}=e^{-2 \varphi} g$

$$
i\left(g^{\prime}\right):=\int_{M} R_{g^{\prime}}^{2} e^{-\varphi} \mathrm{d} V_{g^{\prime}}
$$

As one can easily check, if two metrics $g_{1}$ and $g_{2}$ are homothetic, then $i\left(g_{1}\right)=$ $i\left(g_{2}\right)$. So, we have

$$
I(M, \partial M, g)=\underset{g^{\prime}=e^{-2 \varphi} g}{ } \inf _{\operatorname{Vol}_{g}^{\prime}(M)=1 \text { and }\left|\nabla_{g} \varphi\right|_{g} \leq C_{1}, H_{g^{\prime}}=0}\left(\int_{M} R_{g^{\prime}}^{2} e^{-\varphi} \mathrm{d} V_{g^{\prime}}\right) .
$$

Concerning $I(M, \partial M, g)$, we have the following lemma.

Lemma 3.4. There exists a positive constant $C=C\left(\left\|\nabla R m_{g}\right\|\right)$ such that

$$
I(M, \partial M, g) \geq C(Y(M, \partial M,[g]))^{2} .
$$

Proof. As we have seen

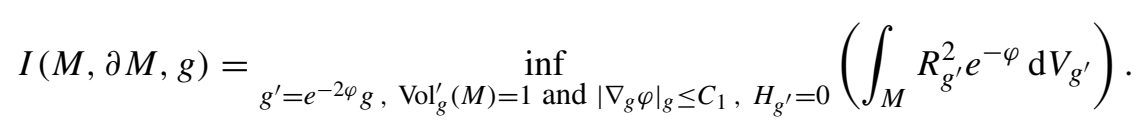

Take $\varphi \in C^{\infty}(M)$ such that, for $g^{\prime}=e^{-2 \varphi} g, \operatorname{Vol}_{g}^{\prime}(M)=1$ and such that $\left|\nabla_{g} \varphi\right|_{g} \leq$ $C_{1}$ where $C_{1}$ is given by Proposition 3.3. Since $\operatorname{Vol}_{g}^{\prime}(M)=1$, if $p$ is a point where $\varphi$ attains its minimum we have

$$
e^{-3 \varphi(p)} \operatorname{Vol}_{g}(M) \geq 1,
$$

and then, there exists $C_{0}$ depending only on $(M, g)$ such that $\varphi(p) \leq C_{0}$. Now, using the mean value theorem, it follows since $\left|\nabla_{g} \varphi\right|_{g}$ is controlled by a constant 
depending only on $(M, g)$, that $\max \varphi \leq C_{0}^{\prime}$ where $C_{0}^{\prime}$ depends only on $(M, g)$. Using this, we clearly have that

$$
\int_{M} R_{g^{\prime}}^{2} e^{-\varphi} \mathrm{d} V_{g^{\prime}} \geq e^{-C_{0}^{\prime}} \int_{M} R_{g^{\prime}}^{2} \mathrm{~d} V_{g^{\prime}} .
$$

Using Hölder inequality and the definition of the Yamabe invariant, since $H_{g^{\prime}}=0$, we get (recall that $\operatorname{Vol}_{g}^{\prime}(M)=1$ )

$$
\int_{M} R_{g^{\prime}}^{2} \mathrm{~d} V_{g^{\prime}} \geq(Y(M, \partial M,[g]))^{2},
$$

and then $I(M, \partial M, g) \geq e^{-C_{0}^{\prime}}(Y(M, \partial M,[g]))^{2}$. This ends the proof.

We will prove a lower bound for a solution to the equation (3.2) following in [9, section 3]. Since we are dealing with manifolds with boundary we have to compute the conformal deformation of the integral of $\sigma_{2}$ in this context. Here is the formula.

Lemma 3.5. For a conformal metric $\tilde{g}=e^{-2 u} g$, we have the following integral transformation

$$
\begin{aligned}
\int_{M} \sigma_{2}\left(\tilde{g}^{-1} A_{\tilde{g}}^{1}\right) e^{-4 u} \mathrm{~d} V_{g}= & \int_{M} \sigma_{2}\left(g^{-1} A_{g}^{1}\right) \mathrm{d} V_{g}+\frac{1}{8} \int_{M} R_{g}\left|\nabla_{g} u\right|_{g}^{2} \mathrm{~d} V_{g} \\
& -\frac{1}{4} \int_{M}\left|\nabla_{g} u\right|_{g}^{4} \mathrm{~d} V_{g}+\frac{1}{2} \int_{M} \Delta_{g} u\left|\nabla_{g} u\right|_{g}^{2} \mathrm{~d} V_{g} \\
& -\frac{1}{2} \int_{M} A_{g}^{1}\left(\nabla_{g} u, \nabla_{g} u\right) \mathrm{d} V_{g} \\
& +\frac{1}{4} \oint_{\partial M} \partial_{\nu} u\left(R_{g}+2 \Delta_{g} u-2\left|\nabla_{g} u\right|_{g}^{2}\right) \mathrm{d} S_{g} \\
& -\oint_{\partial M} A_{g}^{1}\left(\nu, \nabla_{g} u\right) \mathrm{d} S_{g}-\frac{1}{4} \oint_{\partial M} \partial_{\nu}\left|\nabla_{g} u\right|_{g}^{2} \mathrm{~d} S_{g} .
\end{aligned}
$$

In particular, if the boundary of $M$ is totally geodesic and $\partial_{\nu} u=0$, we get

$$
\begin{aligned}
\int_{M} \sigma_{2}\left(\tilde{g}^{-1} A_{\tilde{g}}^{1}\right) e^{-4 u} \mathrm{~d} V_{g}= & \int_{M} \sigma_{2}\left(g^{-1} A_{g}^{1}\right) \mathrm{d} V_{g}+\frac{1}{8} \int_{M} R_{g}\left|\nabla_{g} u\right|_{g}^{2} \mathrm{~d} V_{g} \\
& -\frac{1}{4} \int_{M}\left|\nabla_{g} u\right|_{g}^{4} \mathrm{~d} V_{g}+\frac{1}{2} \int_{M} \Delta_{g} u\left|\nabla_{g} u\right|_{g}^{2} \mathrm{~d} V_{g} \\
& -\frac{1}{2} \int_{M} A_{g}^{1}\left(\nabla_{g} u, \nabla_{g} u\right) \mathrm{d} V_{g} .
\end{aligned}
$$

Proof. For the computations, we will follow in [9, Section 3]. The final formula will be the same as in [9], but with some extra terms coming from the boundary. 
We have

Denote $\tilde{\sigma}_{1}=\sigma_{1}\left(\tilde{g}^{-1} A_{\tilde{g}}^{1}\right), \sigma_{1}=\sigma_{1}\left(g^{-1} A_{g}^{1}\right), \tilde{\sigma_{2}}=\sigma_{2}\left(\tilde{g}^{-1} A_{\tilde{g}}^{1}\right), \sigma_{2}=\sigma_{2}\left(g^{-1} A_{g}^{1}\right)$.

$$
2 \tilde{\sigma}_{2}=\tilde{\sigma}_{1}^{2}-\left|A_{\tilde{g}}^{1}\right|_{\tilde{g}}^{2} \text {. }
$$

By equation (2.3), we have

$$
\tilde{\sigma_{1}} e^{-2 u}=\sigma_{1}+\Delta_{g} u-\frac{1}{2}\left|\nabla_{g} u\right|_{g}^{2},
$$

so

$$
{\tilde{\sigma_{1}}}^{2} e^{-4 u}=\sigma_{1}^{2}+\left(\Delta_{g} u\right)^{2}+\frac{1}{4}\left|\nabla_{g} u\right|_{g}^{4}+2 \sigma_{1} \Delta_{g} u-\Delta_{g} u\left|\nabla_{g} u\right|_{g}^{2}-\sigma_{1}\left|\nabla_{g} u\right|_{g}^{2} .
$$

After an easy computation, we get

$$
\begin{aligned}
\left|A_{\tilde{g}}^{1}\right|_{\tilde{g}}^{2} e^{-4 u}= & \left|A_{g}^{1}\right|_{g}^{2}+\left|\nabla_{g}^{2} u\right|_{g}^{2}+\frac{3}{4}\left|\nabla_{g} u\right|_{g}^{4}-\sigma_{1}\left|\nabla_{g} u\right|_{g}^{2}-\Delta_{g} u\left|\nabla_{g} u\right|_{g}^{2} \\
& +2\left(A_{g}^{1}\right)_{i j} \nabla_{g}^{2 i j} u+2\left(A_{g}^{1}\right)_{i j} \nabla_{g}^{i} u \nabla_{g}^{j} u+2 \nabla_{g i j}^{2} u \nabla_{g}^{i} u \nabla_{g}^{j} u .
\end{aligned}
$$

Putting all together, we obtain

$$
\begin{aligned}
2{\tilde{\sigma_{2}}} e^{-4 u}= & 2 \sigma_{2}+\left(\Delta_{g} u\right)^{2}-\left|\nabla_{g}^{2} u\right|_{g}^{2}-\frac{1}{2}\left|\nabla_{g} u\right|_{g}^{4}+2 \sigma_{1} \Delta_{g} u \\
& -2\left(A_{g}^{1}\right)_{i j} \nabla_{g}^{2 i j} u-2\left(A_{g}^{1}\right)_{i j} \nabla_{g}^{i} u \nabla_{g}^{j} u-2 \nabla_{g}^{2} u \nabla_{g}^{i} u \nabla_{g}^{j} u .
\end{aligned}
$$

Now, by simple computation, we have the following identities

$$
\begin{aligned}
-2 \int_{M}\left(A_{g}^{1}\right)_{i j} \nabla_{g}^{2 i j} u \mathrm{~d} V_{g}= & -2 \int_{M} \sigma_{1} \Delta_{g} u \mathrm{~d} V_{g}+2 \oint_{\partial M} \partial_{\nu} u \sigma_{1} \mathrm{~d} S_{g} \\
& -2 \oint_{\partial M} A_{g}^{1}\left(\nu, \nabla_{g} u\right) \mathrm{d} S_{g} \\
-2 \int_{M} \nabla_{i j}^{2} u \nabla_{g}^{i} u \nabla_{g}^{j} u \mathrm{~d} V_{g}= & \int_{M} \Delta_{g} u\left|\nabla_{g} u\right|_{g}^{2} \mathrm{~d} V_{g}-\oint_{\partial M} \partial_{\nu} u\left|\nabla_{g} u\right|_{g}^{2} \mathrm{~d} S_{g},
\end{aligned}
$$

where we integrated by parts and we used the Schur's lemma,

$$
2 \nabla_{g}^{j}\left(\operatorname{Ric}_{g}\right)_{i j}=\nabla_{i} R_{g},
$$

for the first identity. Finally we get

$$
\begin{aligned}
2 \int_{M} \tilde{\sigma_{2}} e^{-4 u} \mathrm{~d} V_{g}= & 2 \int_{M} \sigma_{2} \mathrm{~d} V_{g} \\
& +\int_{M}\left[\left(\Delta_{g} u\right)^{2}-\left|\nabla_{g}^{2} u\right|_{g}^{2}-\frac{1}{2}\left|\nabla_{g} u\right|_{g}^{4}+\Delta_{g} u\left|\nabla_{g} u\right|_{g}^{2}\right. \\
& \left.-2 A_{g}^{1}\left(\nabla_{g} u, \nabla_{g} u\right)\right] \mathrm{d} V_{g} \\
& +\oint_{\partial M} \partial_{\nu} u\left(\frac{1}{2} R_{g}-2 A_{g}^{1}\left(\nu, \nabla_{g} u\right)-\left|\nabla_{g} u\right|_{g}^{2}\right) \mathrm{d} S_{g},
\end{aligned}
$$


Now, integrating the Bochner formula

$$
\frac{1}{2} \Delta_{g}\left|\nabla_{g} u\right|_{g}^{2}=\left|\nabla_{g}^{2} u\right|_{g}^{2}+R i c_{g}\left(\nabla_{g} u, \nabla_{g} u\right) \mathrm{d} V_{g}+\nabla_{i} u, \nabla^{i}\left(\Delta_{g} u\right),
$$

we get

$$
\begin{aligned}
\frac{1}{2} \oint_{\partial M} \partial_{\nu}\left|\nabla_{g} u\right|_{g}^{2} \mathrm{~d} S_{g}= & \int_{M}\left[\left|\nabla_{g}^{2} u\right|_{g}^{2}-\left(\Delta_{g} u\right)^{2}+\operatorname{Ric}_{g}\left(\nabla_{g} u, \nabla_{g} u\right)\right] \mathrm{d} V_{g} \\
& +\oint_{\partial M} \partial_{\nu} u \Delta_{g} u \mathrm{~d} S_{g} .
\end{aligned}
$$

Using the definition of the Schouten tensor $A_{g}^{1}$, we get the first point of the lemma.

Now, if the boundary is totally geodesic and $\partial_{v} u=0$ on $\partial M$, then by Lemma 2.10 we have that all the boundary terms must vanish. Thus the second point of the lemma is proved. This completes the proof.

Since $(M, g)$ has totally geodesic boundary, the boundary terms don't effect the conformal transformation of the integral of $\sigma_{2}$. Hence, following in $[9$, Section 3] and using Lemma 3.4, we obtain the lower bound.

Proposition 3.6 (Lower Bound). Assume that for some $t \in\left[\delta, t_{0}\right], t_{0} \leq 2 / 3$, the following estimate holds

$$
\int_{M} \sigma_{2}\left(g^{-1} A_{g}^{1}\right) \mathrm{d} V_{g}+C\left(\frac{7}{10}-t\right)\left(Y(M, \partial M,[g])^{2}=\mu_{t}>0,\right.
$$

for some $C$ depending only on $\left\|\nabla R m_{g}\right\|$. Then there exists $\underline{\delta}$ depending only on $\operatorname{diam}_{g}(M)$ and $\left\|\nabla R m_{g}\right\|$ such that if $u_{t} \in C^{2}(M)$ is a solution of (3.2) and if $A_{u_{t}}^{t} \in \Gamma_{2}^{+}$then $u_{t} \geq \underline{\delta}$.

We have the following $C^{2, \alpha}$ estimate for solutions of the equation (3.2).

Proposition 3.7 ( $C^{2, \alpha}$ estimate). Let $u_{t} \in C^{4}(M)$ be a solution of (3.2) for some $\delta \leq t \leq t_{0}, t_{0} \leq 2 / 3$, satisfying $\underline{\delta}<u_{t}<\bar{\delta}$, and $\left\|\nabla u_{t}\right\|_{g, \infty}<C_{1}$. Then, if $A_{u_{t}}^{t} \in \Gamma_{2}^{+}$, for $0<\alpha<1,\left\|u_{t}\right\|_{C^{2, \alpha}} \leq C_{2}$, where $C_{2}$ depends only on $\underline{\delta}, \bar{\delta}, C_{1}$ and $\left\|\nabla^{2} R m_{g}\right\|$.

Proof. The interior $C^{2}$ estimate follows from the work of Chen [11] and the boundary $C^{2}$ estimate follows from [12, Theorem $\left.6(\mathrm{~b})\right]$. With the $C^{2}$ estimate at hand, we obtain high-order estimate (in particular $C^{2, \alpha}$ one) from the works of Evans [19], Krylov [28] and Lions-Trudinger [29].

Since we proved $C^{2, \alpha}$ estimates for solutions of the equation (3.2), by the classical Ascoli-Arzela's Theorem, we have that $\mathcal{S}$ is closed, therefore $\mathcal{S}=\left[\delta, t_{0}\right]$. In particular $t_{0} \in \mathcal{S}$. Hence the metric 
$\tilde{g}=e^{-2 u_{t_{0}}} g$ then satisfies $\sigma_{2}\left(A_{\tilde{g}}^{t_{0}}\right)>0, R_{\tilde{g}}>0$ and $L_{\tilde{g}}=0$. Furthermore, by Lemma 2.5 we have that the metric $\tilde{g}$ satisfies

$$
\left(3 t_{0}-2\right) R_{\tilde{g}} \tilde{g}<6 \operatorname{Ric}_{\tilde{g}}<3\left(2-t_{0}\right) R_{\tilde{g}} \tilde{g} .
$$

Hence the proof of Theorem 3.1 is complete.

Now we are going to give the proof of Theorem 1.7.

Proof of Theorem 1.7. First of all from $R_{g}>0$ and $L_{g}=0$, we infer $Y(M, \partial M$, $[g])>0$. On the other hand, one can easily check that

$$
\sigma_{2}\left(g^{-1} A_{g}\right)=\frac{3}{16}\left|R_{g}\right|^{2}-\frac{1}{2}\left|\operatorname{Ric}_{g}\right|^{2} .
$$

Thus, we have $\int_{M} \sigma_{2}\left(g^{-1} A_{g}\right) \geq 0$ is equivalent to $\int_{M}\left|\operatorname{Ric}_{g}\right|^{2} \mathrm{~d} V_{g} \leq \frac{3}{8} \int_{M}\left|R_{g}\right|^{2} \mathrm{~d} V_{g}$. Hence we can apply Theorem 3.1 with $t_{0}=\frac{2}{3}$ and get the existence of a metric $\tilde{g}$ conformal to $g$ such that $\operatorname{Ric}_{\tilde{g}}>0$ and $L_{\tilde{g}}=0$. Hence appealing to Theorem 1.5, we have the proof of Theorem 1.7 is complete.

\section{Four manifolds with boundary}

In this section, we give the proof of Theorem 1.8. As for the case of 3-manifolds, we are going to prove a more general theorem from which Theorem 1.8 becomes a direct application.

Theorem 4.1. Let $(M, g)$ be a compact four-dimensional Riemannian manifold with umbilic boundary and $0 \leq \alpha \leq 1$. If $Y(M, \partial M,[g])>0$, and

$$
\frac{1}{2} \kappa_{\left(P^{4}, P^{3}\right)}-\frac{\alpha}{16} \int_{M}\left|W_{g}\right|_{g}^{2} \mathrm{~d} V_{g}+\frac{1}{24}\left(1-t_{0}\right)\left(2-t_{0}\right) Y(M, \partial M,[g])^{2}>0,
$$

for some $t_{0} \leq 1$, then there exists a conformal metric $\tilde{g}=e^{-2 u} g$ whose curvature satisfies

$$
R_{\tilde{g}}>0, \quad \sigma_{2}\left(\tilde{g}^{-1} A_{\tilde{g}}^{t_{0}}\right)-\frac{\alpha}{16}\left|W_{\tilde{g}}\right|_{\tilde{g}}^{2}>0, \text { and } H_{\tilde{g}}=0 .
$$

This implies the pointwise inequalities

$$
\left(t_{0}-1\right) R_{\tilde{g}} \tilde{g}<2 R i c_{\tilde{g}}<\left(2-t_{0}\right) R_{\tilde{g}} \tilde{g} .
$$

Throughout the sequel, $(M, g)$ will be a compact 4-dimensional Riemannian manifold with umbilic boundary and with positive Yamabe invariant $Y(M, \partial M,[g])$. Since all the hypothesis on the metric $g$ are conformally invariant, then by a result of Escobar, see [18], we can choose in the conformal class the Yamabe metric, i.e. a metric with positive constant scalar curvature and zero mean curvature. Moreover, since umbilicity is also conformally invariant, we have that the boundary must be 
totally geodesic. Hence, from now on, $(M, g)$ will be a compact four-manifold with totally geodesic boundary, positive constant scalar curvature and satisfying the integral pinching condition.

On the other hand, since $M$ is compact and $R_{g}>0$, there exist $t_{0}>\delta>-\infty$, $\delta<0$ such that $A_{g}^{\delta}$ is positive definite (i.e. Ric $-\frac{\delta}{6} R>0$ on $M$ ). Moreover we can choose $\delta$ so small such that

$$
\sigma_{2}^{1 / 2}\left(g^{-1} A_{g}^{\delta}\right)-\frac{\sqrt{\alpha}}{4}\left|W_{g}\right|_{g}>0 .
$$

Note that $\delta$ depends only on $\|R m\|$.

Now we define a subclass of the positive cone of order 2 which will be useful in our arguments:

Definition 4.2. For a conformal metric $\tilde{g}=e^{-2 u} g$, we define the set

$$
\Lambda_{\tilde{g}}^{+}=\left\{t \in\left[\delta, t_{0}\right] \mid A_{\tilde{g}}^{t} \in \Gamma_{2}^{+} \text {and } \sigma_{2}^{1 / 2}\left(g^{-1} A_{\tilde{g}}^{t}\right)-\frac{\sqrt{\alpha}}{4}\left|W_{g}\right|_{g}>0\right\} \text {. }
$$

In particular if $t \in \Lambda_{\tilde{g}}^{+}$then $A_{\tilde{g}}^{t} \in \Gamma_{2}^{+}$.

We point out that $\delta \in \Lambda_{g}^{+}$.

For $t \in\left[\delta, t_{0}\right]$, consider the path of equations (in the sequel we use the notation $A_{u_{t}}^{t}:=A_{g_{t}}^{t}$ for $g_{t}$ given by $g_{t}=e^{-2 u_{t}} g$ )

$$
\begin{cases}\sigma_{2}^{1 / 2}\left(g^{-1} A_{u_{t}}^{t}\right)-\frac{\sqrt{\alpha}}{4}\left|W_{g}\right|_{g}=f e^{2 u_{t}} & \text { in } M \\ \partial_{\nu} u=0 & \text { on } \partial M\end{cases}
$$

where $f(x)=\sigma_{2}^{1 / 2}\left(g^{-1} A_{g}^{\delta}\right)-\frac{\sqrt{\alpha}}{4}\left|W_{g}\right|_{g}>0$. Note that $u \equiv 0$ is a solution of (4.1) for $t=\delta$.

As for the tree-dimensional case, we use the continuity method. Define

$$
\mathcal{S}=\left\{t \in\left[\delta, t_{0}\right] \mid \exists \text { a solution } u_{t} \in C^{2, \alpha}(M) \text { of (4.1) with } t \in \Lambda_{u_{t}}^{+}\right\}
$$

Clearly, with our choice of $f, u \equiv 0$ is a solution for $t=\delta$. Since $\delta \in \Lambda_{g}^{+}$, then $\delta \in \mathcal{S}$. Hence, we have $\mathcal{S} \neq \emptyset$. Let $t \in \mathcal{S}$, and $u_{t}$ be a solution. By Proposition 2.6, the linearized operator at $u_{t}, \mathcal{L}^{t}: C^{2, \alpha}(M) \cap\left\{\partial_{\nu} u=0\right.$ on $\left.\partial M\right\} \rightarrow C^{\alpha}(M)$, is invertible (note that the additional term in the right hand side of the equation does not effect linearization). The implicit function theorem tells us that $\mathcal{S}$ is open. To prove that $\mathcal{S}$ is close we need to establish a priori $C^{2, \alpha}$ estimates for solutions of the equation (4.1). To do so, we start by establishing upper-bound estimate as for the case of 3-manifolds.

Proposition 4.3 (Upper bound). Let $u_{t} \in C^{2}(M)$ be a solution of (4.1) for some $t \in\left[\delta, t_{0}\right]$, with $t \in \Lambda_{u_{t}}^{+}$. Then $u_{t} \leq \bar{\delta}$, where $\bar{\delta}$ depends only on $\left\|R m_{g}\right\|$. 
Proof. From Lemma 2.3 (iv), we have $\frac{4}{\sqrt{6}} \sigma_{2}^{1 / 2} \leq \sigma_{1}$, so for all $p \in M$

$$
\frac{4}{\sqrt{6}} \frac{\sqrt{\alpha}}{4}\left|W_{g}\right|_{g}+\frac{4}{\sqrt{6}} f e^{2 u_{t}} \leq \sigma_{1}\left(g^{-1} A_{u_{t}}^{t}\right) \text {. }
$$

Let $p \in M$ be the maximum of $u_{t}$, then (this is true also if $p \in \partial M$, since $\partial_{\nu} u=0$ on $\partial M)$ we have $\left(\Delta u_{t}\right)(p) \leq 0$. Then, using (2.2), we have

$$
\begin{aligned}
\frac{4}{\sqrt{6}} \frac{\sqrt{\alpha}}{4}\left(\left|W_{g}\right|_{g}\right)(p)+\frac{4}{\sqrt{6}} f(p) e^{2 u_{t}(p)} & \leq \sigma_{1}\left(g^{-1} A_{u_{t}}^{t}\right)(p) \\
& =\sigma_{1}\left(g^{-1} A_{g}^{t}\right)(p)+(3-2 t)\left(\Delta u_{t}\right)(p) \\
& \leq \sigma_{1}\left(g^{-1} A_{g}^{t}\right)(p) \\
& \leq \sigma_{1}\left(g^{-1} A_{g}^{\delta}\right)(p)
\end{aligned}
$$

This implies

$$
\frac{4}{\sqrt{6}} f(p) e^{2 u_{t}(p)} \leq \sigma_{1}\left(g^{-1} A_{g}^{\delta}\right)(p)-\frac{4}{\sqrt{6}} \frac{\sqrt{\alpha}}{4}\left(\left|W_{g}\right|_{g}\right)(p),
$$

where the last term has positive sign. Since $M$ is compact, this implies $u_{t} \leq \bar{\delta}$, for some $\bar{\delta}$ depending only on $\|R m\|$.

Following the previous section, once we have an upper bound of the solution, from Proposition 3.3, we get gradient estimates. Now we are going to establish the lower-bound estimates. To do that we need the following lemma.

Lemma 4.4. If $\hat{g}$ is a Riemannian metric on $M$ conformal to $g$ such that $L_{\hat{g}}=0$, then

$$
\int_{M} \sigma_{2}\left(\hat{g}^{-1} A_{\hat{g}}\right)=\frac{1}{2} \kappa_{\left(P^{4}, P^{3}\right)} .
$$

Proof. First of all, one can easily check that the following holds

$$
Q_{\hat{g}}=2 \sigma_{2}\left(\hat{g}^{-1} A_{\hat{g}}\right)-\frac{1}{12} \Delta_{\hat{g}} R_{\hat{g}}
$$

Thus integrating this equation and using the divergence theorem, we get

$$
\int_{M} Q_{\hat{g}} \mathrm{~d} V_{\hat{g}}=2 \int_{M} \sigma_{2}\left(\hat{g}^{-1} A_{\hat{g}}\right) \mathrm{d} V_{\hat{g}}+\frac{1}{12} \oint_{\partial M} \frac{\partial R_{\hat{g}}}{\partial n_{\hat{g}}} \mathrm{~d} S_{\hat{g}} .
$$

On the other hand, since $L_{\hat{g}}=0$, then

$$
T_{\hat{g}}=-\frac{1}{12} \frac{\partial R_{\hat{g}}}{\partial n_{\hat{g}}} .
$$


Thus we obtain

$$
\int_{M} Q_{\hat{g}} \mathrm{~d} V_{\hat{g}}=2 \int_{M} \sigma_{2}\left(\hat{g}^{-1} A_{\hat{g}}\right) \mathrm{d} V_{\hat{g}}-\oint_{\partial M} T_{\hat{g}} \mathrm{~d} S_{\hat{g}}
$$

Hence, we get

$$
\int_{M} \sigma_{2}\left(\hat{g}^{-1} A_{\hat{g}}\right)=\frac{1}{2} \kappa_{\left(P^{4}, P^{3}\right)} .
$$

This completes the proof of the lemma.

Proposition 4.5 (Lower bound). Assume that for some $t \in\left[\delta, t_{0}\right]$ the following estimate holds

$$
\frac{1}{2} \kappa_{\left(P^{4}, P^{3}\right)}-\frac{\alpha}{16} \int_{M}\left|W_{g}\right|_{g}^{2} \mathrm{~d} V_{g}+\frac{1}{24}(1-t)(2-t) Y(M, \partial M,[g])^{2}=\mu_{t}>0 .
$$

Then there exist $\underline{\delta}$ depending only on $\operatorname{diam}(M, g)$ and $\left\|\nabla^{2} R m\right\|$ such that if $u_{t} \in$ $C^{2}(M)$ is a solution of (4.1) and if $t \in \Lambda_{u_{t}}^{+}$then $u_{t} \geq \underline{\delta}$.

Proof. Since $A_{g}^{t}=A_{g}^{1}+\frac{1-t}{2} \sigma_{1}\left(A_{g}^{1}\right) g$, we easily have

$$
\sigma_{2}\left(A_{g}^{t}\right)=\sigma_{2}\left(A_{g}^{1}\right)+\frac{3}{2}(1-t)(2-t) \sigma_{1}\left(A_{g}^{1}\right)^{2}
$$

Letting $\tilde{g}=e^{-2 u_{t}} g$, since $u_{t}$ is a solution of equation (4.1), we have

$$
f^{2} e^{4 u_{t}}+\frac{\sqrt{\alpha}}{2} f\left|W_{g}\right|_{g} e^{2 u_{t}}=\sigma_{2}\left(g^{-1} A_{u_{t}}^{t}\right)-\frac{\alpha}{16}\left|W_{g}\right|_{g}^{2}
$$

The left-hand side can be estimated by

$$
f^{2} e^{4 u_{t}}+\frac{\sqrt{\alpha}}{2} f\left|W_{g}\right|_{g} e^{2 u_{t}} \leq C^{\prime} e^{2 u_{t}}
$$

where the positive constant $C^{\prime}$ depends only on $\|R m\|$. So we get

$$
\begin{aligned}
C^{\prime} e^{2 u_{t}} & \geq \sigma_{2}\left(g^{-1} A_{u_{t}}^{t}\right)-\frac{\alpha}{16}\left|W_{g}\right|_{g}^{2} \\
& =e^{-4 u_{t}}\left(\sigma_{2}\left(\tilde{g}^{-1} A_{u_{t}}^{1}\right)+\frac{1}{24}(1-t)(2-t) R_{\tilde{g}}^{2}\right)-\frac{\alpha}{16}\left|W_{g}\right|_{g}^{2}
\end{aligned}
$$


Integrating this with respect to $\mathrm{d} V_{g}$, we obtain

$$
\begin{aligned}
C^{\prime} \int_{M} e^{2 u_{t}} \mathrm{~d} V_{g} \geq & \int_{M} \sigma_{2}\left(\tilde{g}^{-1} A_{u_{t}}^{1}\right) \mathrm{d} V_{\tilde{g}}-\frac{\alpha}{16} \int_{M}\left|W_{g}\right|_{g}^{2} \mathrm{~d} V_{g} \\
& +\frac{1}{24}(1-t)(2-t) \int_{M} R_{\tilde{g}}^{2} \mathrm{~d} V_{\tilde{g}} \\
= & \frac{1}{2} \kappa_{\left(P^{4}, P^{3}\right)}-\frac{\alpha}{16} \int_{M}\left|W_{g}\right|_{g}^{2} \mathrm{~d} V_{g}+\frac{1}{24}(1-t)(2-t) \int_{M} R_{\tilde{g}}^{2} \mathrm{~d} V_{\tilde{g}} \\
\geq & \frac{1}{2} \kappa_{\left(P^{4}, P^{3}\right)}-\frac{\alpha}{16} \int_{M}\left|W_{g}\right|_{g}^{2} \mathrm{~d} V_{g} \\
& +\frac{1}{24}(1-t)(2-t) Y(M, \partial M,[g])^{2}=\mu_{t}>0,
\end{aligned}
$$

where we have used Lemma 4.4, and the fact that for any conformal metric $g^{\prime} \in[g]$, if $H_{g^{\prime}}=0$, then

$$
\int_{M} R_{g^{\prime}}^{2} \mathrm{~d} V_{g^{\prime}} \geq Y(M, \partial M,[g])^{2} .
$$

This gives

$$
\max _{M} u_{t} \geq \log \mu_{t}-C(\operatorname{diam}(M, g),\|R m\|) .
$$

Since, as already remarked $\max _{M}\left|\nabla_{g} u_{t}\right|_{g} \leq C_{1}$ by the same arguments as the ones of Proposition 3.3, then we have the Harnack inequality

$$
\max _{M} u_{t} \leq \min _{M} u_{t}+C\left(\operatorname{diam}(M, g),\left\|\nabla^{2} R m\right\|\right),
$$

by simply integrating along a geodesic connecting points at witch $u_{t}$ attains its maximum and minimum. Combining this two inequalities, we obtain

$$
u_{t} \geq \min _{M} u_{t} \geq \log \mu_{t}-C=: \underline{\delta},
$$

where $C$ depends only on $\operatorname{diam}(M, g)$ and $\left\|\nabla^{2} R m\right\|$.

Once we have $C^{0}$ and $C^{1}$ estimates, using the same arguments as the ones of Proposition 3.7, we get $C^{2, \alpha}$ estimates. Thus we are ready to apply the continuity method as in the 3-dimensional case, and conclude the proof of Theorem 4.1.

Now we are ready to present the proof of Theorem 1.8.

Proof of Theorem 1.8. First of all, since $Y(M, \partial M,[g])>0$, and $\kappa_{\left(P^{4}, P^{3}\right)}>$ $\frac{1}{8} \int_{M}\left|W_{g}\right|^{2} \mathrm{~d} V_{g}$, then we can apply Theorem 4.1 with $t_{0}=1$ and $\alpha=1$ and get the existence of a metric $\tilde{g}$ conformal to $g$ such that

$$
\sigma_{2}\left(g^{-1} A_{\tilde{g}}\right)>\frac{1}{16}\left|W_{g}\right|^{2}, \quad \text { and } \quad L_{\tilde{g}}=0 .
$$


This is equivalent to

$$
\sigma_{2}\left(\tilde{g}^{-1} A_{\tilde{g}}\right)>\frac{1}{16}\left|W_{\tilde{g}}\right|^{2} \text {, and } L_{\tilde{g}}=0 .
$$

On the other hand, one can check easily that the following holds

$$
\sigma_{2}\left(\tilde{g}^{-1} A_{\tilde{g}}\right)=\frac{1}{96} R_{\tilde{g}}^{2}-\frac{1}{8}\left|E_{\tilde{g}}\right|^{2} .
$$

Thus, we obtain

$$
\frac{1}{6} R_{\tilde{g}}^{2}-2\left|E_{\tilde{g}}\right|^{2}>\left|W_{\tilde{g}}\right|^{2} .
$$

So rearranging the latter inequality, we get the Margerin's weak pinching condition, namely

$$
W P_{\tilde{g}}<\frac{1}{6} .
$$

Hence, applying Theorem 1.6, we conclude the proof of Theorem 1.8.

\section{Principal eigenvalue of $P_{g}^{4,3}$ and applications}

In this section, we provide the proof of Theorem 1.9 and Corollary 1.10. We start by giving a proposition which will be used for the proof of Theorem 1.9.

Proposition 5.1. Let $(M, g)$ be a compact four-dimensional Riemannian manifold with boundary such that $L_{g}=0$. Assuming Ric $c_{g} \leq R_{g} g$, then we have $P_{g}^{4,3}$ is a non-negative operator and $\operatorname{ker} P_{g}^{4,3} \simeq R$.

Proof. First of all, since $L_{g}=0$, then for every $u \in H_{\frac{\partial}{\partial n}}$, we have

$$
\left\langle P_{g}^{4,3} u, u\right\rangle_{L^{2}(M)}=\int_{M}\left(\Delta_{g} u\right)^{2} \mathrm{~d} V_{g}+\frac{2}{3} \int_{M} R_{g}\left|\nabla_{g} u\right|^{2} \mathrm{~d} V_{g}-2 \int_{M} \operatorname{Ric}_{g}\left(\nabla_{g} u, \nabla_{g} u\right) \mathrm{d} V_{g} .
$$

Now we recall the Bochner identity

$$
\frac{1}{2} \Delta_{g}\left(\left|\nabla_{g} u\right|^{2}\right)=\left|\nabla_{g}^{2} u\right|^{2}+\operatorname{Ric}_{g}\left(\nabla_{g} u, \nabla_{g} u\right)+<\nabla_{g} u, \nabla_{g}\left(\Delta_{g} u\right)>.
$$

Integrating the latter formula, applying the divergence theorem and integrating by parts, we get

$$
\begin{aligned}
-\frac{1}{2} \oint_{\partial M} \frac{\partial\left(\left|\nabla_{g} u\right|^{2}\right)}{\partial n_{g}} \mathrm{~d} S_{g}= & \int_{M}\left|\nabla_{g}^{2} u\right|^{2} \mathrm{~d} V_{g}+\int_{M} \operatorname{Ric}_{g}\left(\nabla_{g} u, \nabla_{g} u\right)-\int_{M}\left(\Delta_{g} u\right)^{2} \mathrm{~d} V_{g} \\
& -\oint_{\partial M} \frac{\partial u}{\partial n_{g}} \Delta_{g} u \mathrm{~d} S_{g} .
\end{aligned}
$$


Recalling that $u \in H_{\frac{\partial}{\partial n}}$, then

$$
\oint_{\partial M} \frac{\partial u}{\partial n_{g}} \Delta_{g} u \mathrm{~d} S_{g}=0
$$

Using Lemma 2.10, we obtain

$$
\frac{\partial\left(\left|\nabla_{g} u\right|^{2}\right)}{\partial n_{g}}=0
$$

Hence, we get

$$
\int_{M}\left|\nabla_{g}^{2} u\right|^{2} \mathrm{~d} V_{g}+\int_{M} \operatorname{Ric}_{g}\left(\nabla_{g} u, \nabla_{g} u\right)=\int_{M}\left(\Delta_{g} u\right)^{2} \mathrm{~d} V_{g} .
$$

Now, using the latter formula, we have

$$
\begin{aligned}
\left\langle P_{g}^{4,3} u, u\right\rangle_{L^{2}(M)}= & -\frac{1}{3} \int_{M}\left(\Delta_{g} u\right)^{2} \mathrm{~d} V_{g}+\frac{4}{3} \int_{M}\left|\nabla_{g}^{2} u\right|^{2} \mathrm{~d} V_{g}+\frac{2}{3} \int_{M} R_{g}\left|\nabla_{g} u\right|^{2} \mathrm{~d} V_{g} \\
& -\frac{2}{3} \int_{M} \operatorname{Ric}_{g}\left(\nabla_{g} u, \nabla_{g} u\right) \mathrm{d} V_{g} .
\end{aligned}
$$

Next, setting

$$
\bar{\nabla}_{g}^{2} u=\nabla_{g}^{2} u-\frac{1}{4} \Delta_{g} g
$$

we get

$$
\left\langle P_{g}^{4,3} u, u\right\rangle_{L^{2}(M)}=\frac{4}{3} \int_{M}\left|\bar{\nabla}_{g}^{2} u\right|^{2} \mathrm{~d} V_{g}+\frac{2}{3} \int_{M}\left(R_{g} g-\operatorname{Ric}_{g}\right)\left(\nabla_{g} u, \nabla_{g} u\right) \mathrm{d} V_{g} .
$$

So using the hypothesis $R_{g} g-\mathrm{Ric}_{g} \geq 0$, we infer

$$
\left\langle P_{g}^{4,3} u, u\right\rangle_{L^{2}(M)} \geq \frac{4}{3} \int_{M}\left|\bar{\nabla}_{g}^{2} u\right|^{2} \mathrm{~d} V_{g}
$$

Hence, we obtain $P_{g}^{4,3}$ is a non-negative operator. So to finish the proof of the proposition, it remains only to show that the kernel is constituted only by constants. In order to do that, we assume that there exists a non constant function $u \in H_{\frac{\partial}{\partial n}}$ such that $P_{g}^{4,3} u=0$, and argue for a contradiction. From the fact that $u \in \operatorname{ker} P_{g}^{4,3}$, we infer that

$$
\nabla_{g}^{2} u-\frac{1}{4} \Delta_{g} g=0 .
$$

Now calling the doubling of $M$ by $D M$, and the reflected metric by $\bar{g}$, we have that $\bar{g}$ is $C^{2, \alpha}$. Next we reflect $u$ across $\partial M$ and call the reflection by $u_{D M}$. Thus, we obtain an element in $H^{2}(D M)$ verifying

$$
\nabla_{\bar{g}}^{2} u_{D M}-\frac{1}{4} \Delta_{\bar{g}} \bar{g}=0 .
$$


Thus using a result of Tashiro [38], we infer that $(D M, \bar{g})$ is conformally diffeomorphic to $S^{4}$. Thus $(M, g)$ is also conformally diffeomorphic to $S_{+}^{4}$. So we derive the existence of a metric $\tilde{g}$ conformal to $g$ on $M$ which is Einstein, of constant positive scalar curvature, and $L_{\tilde{g}}=0$. Hence using the conformal invariance of $P_{g}^{4,3}$, we get

$$
\frac{4}{3} \int_{M}\left|\bar{\nabla}_{\tilde{g}}^{2} u\right|^{2} \mathrm{~d} V_{\tilde{g}}+\frac{1}{2} R_{\tilde{g}} \int_{M}\left|\nabla_{\tilde{g}} u\right|^{2} \mathrm{~d} V_{\tilde{g}}=0 .
$$

Thus, we obtain $u$ is constant and reach a contradiction. This completes the proof of the proposition.

Having this at hand, we are ready to give the proof of Theorem 1.9.

Proof of Theorem 1.9. Applying Theorem 4.1 with $t_{0}=0$, and $\alpha=0$, we get the existence of a metric $\tilde{g}$ conformal to $g$ such that

$$
\operatorname{Ric}_{\tilde{g}} \leq R_{\tilde{g}} \tilde{g}, \quad \text { and } \quad L_{\tilde{g}}=0 .
$$

Hence appealing to Proposition 5.1, we obtain that $P_{\tilde{g}}^{4,3}$ is non-negative and $\operatorname{ker} P_{\tilde{g}}^{4,3} \simeq \mathbb{R}$. Now recalling that the non-negativity of the operator $P_{g}^{4,3}$ and the triviality of its kernel are conformally invariant properties, we have that the proof of Theorem 1.9 is complete.

Next, we are going to present the proof of Corollary 1.10.

Proof of Corollary 1.10. Due to (1.1), the existence of constant $Q$-curvature, constant $T$-curvature ad zero mean curvature metrics conformal to the background one $g$ is equivalent to solving the following (BVP)

$$
\begin{cases}P_{g}^{4} u+2 Q_{g}=2 \bar{Q} e^{4 u} & \text { in } \quad M \\ P_{g}^{3} u+T_{g}=\bar{T} e^{3 u} & \text { on } \quad \partial M \\ \frac{\partial u}{\partial n_{g}}=0 & \text { on } \partial M .\end{cases}
$$

Where $\bar{Q}$ and $\bar{T}$ are constant real numbers. On the other hand it is easy to see that critical points of the functional

$$
\begin{aligned}
I I(u)= & \left\langle P_{g}^{4,3} u, u\right\rangle_{L^{2}(M)}+4 \int_{M} Q_{g} u \mathrm{~d} V_{g}+\oint_{\partial M} T_{g} u \mathrm{~d} S_{g} \\
& -\kappa_{P_{g}^{4}} \log \int_{M} e^{4 u} \mathrm{~d} V_{g}-\frac{4}{3}\left(\kappa_{\left(P^{4}, P^{3}\right)}-\kappa_{P_{g}^{4}}\right) \log \oint_{\partial M} e^{3 u} \mathrm{~d} S_{g} ;
\end{aligned}
$$

are weak solution of (5.1), hence from standard elliptic regularity theory, they are smooth solutions. Thus to prove the corollary, we will prove the existence of critical 
points. More precisely, under our assumption, we will prove the existence of a minimizer. To do so, we first point out that the functional $I I$ is invariant by translation by constant, and can also be written in the following form

$$
\begin{aligned}
I I(u)= & \left\langle P_{g}^{4,3} u, u\right\rangle_{L^{2}(M)}+4 \int_{M} Q_{g}(u-\bar{u}) \mathrm{d} V_{g}+\oint_{\partial M} T_{g}\left(u-\bar{u}_{\partial M}\right) \mathrm{d} S_{g} \\
& -\kappa_{P_{g}^{4}} \log \int_{M} e^{4(u-\bar{u})} \mathrm{d} V_{g}-\frac{4}{3}\left(\kappa_{\left(P^{4}, P^{3}\right)}-\kappa_{P_{g}^{4}}\right) \log \oint_{\partial M} e^{\left(3 u-\bar{u}_{\partial M}\right)} \mathrm{d} S_{g} .
\end{aligned}
$$

Now exploiting this way of writing $I I$, we have if $\kappa_{P_{g}^{4}} \leq 0$, then by Jensen's inequality, we obtain

$$
I I(u) \geq\left\langle P_{g}^{4,3} u, u\right\rangle_{L^{2}(M)}+4 \int_{M} Q_{g}(u-\bar{u}) \mathrm{d} V_{g}+\oint_{\partial M} T_{g}\left(u-\bar{u}_{\partial M}\right) \mathrm{d} S_{g} .
$$

Hence, using Cauchy inequality, trace theorem, Sobolev embedding, Poincaré inequality, and Lemma 2.9, we get

$$
I I(u) \geq \gamma\|u-\bar{u}\|_{H^{2}}-C
$$

for some $\gamma>0$ and some large $C$. Next if $\kappa_{P_{g}^{4}}>0$, we use Proposition 2.7 and Proposition 2.8 to obtain

$$
\begin{aligned}
I I(u) \geq & \left\langle P_{g}^{4,3} u, u\right\rangle_{L^{2}(M)}+4 \int_{M} Q_{g}(u-\bar{u}) \mathrm{d} V_{g}+\oint_{\partial M} T_{g}\left(u-\bar{u}_{\partial M}\right) \mathrm{d} S_{g} \\
& +\left(-\frac{4}{\alpha_{1}} \kappa_{P_{g}^{4}}-\frac{3}{\alpha_{2}}\left(\kappa_{\left(P^{4}, P^{3}\right)}-\kappa_{P_{g}^{4}}\right)\right)\left\langle P_{g}^{4,3} u, u\right\rangle_{L^{2}(M)}-C_{\alpha_{1}, \alpha_{2}} ;
\end{aligned}
$$

for $\alpha_{1}<16 \pi^{2}$ and $\alpha_{2}<12 \pi^{2}$, and $C_{\alpha, \alpha_{2}}$ a constant depending only on $\alpha_{1}, \alpha_{2}$ and $(M, g)$. To continue the proof we need the following rigidity result.

Lemma 5.2. Let $(M, g)$ be a compact four-dimensional Riemannian manifold with umbilic boundary. Assuming that $Y(M, \partial M,[g]) \geq 0$, we have $\kappa_{\left(P^{4}, P^{3}\right)} \leq 4 \pi^{2}$ and equality holds if and only if $(M, g)$ is conformally diffeomorphic to $S_{+}^{4}$ with its standard metric.

Proof. Since $Y(M, \partial M,[g]) \geq 0$ and $(M, g)$ has umbilic boundary, then by a result of Escobar [18], we can take the Yamabe metric $\tilde{g}$ which has constant non-negative scalar curvature and such that $L_{\tilde{g}}=0$. On the other hand, still by a result of Escobar [18], we have that

$$
Y(M, \partial M,[g])=R_{\tilde{g}} \operatorname{Vol}_{\tilde{g}}(M)^{\frac{1}{2}} \leq Y\left(S_{+}^{4}, S^{3},\left[g_{S}\right]\right)=8 \sqrt{3} \pi ;
$$

and equality holds if and only if $(M, g)$ is conformally diffeomorphic to $\left(S_{+}^{4}, g_{S}\right)$. Now using Lemma 4.4, we have

$$
\kappa_{\left(P^{4}, P^{3}\right)}=2 \int_{M} \sigma_{2}\left(\tilde{g}^{-1} A_{\tilde{g}}\right) \mathrm{d} V_{\tilde{g}}
$$


On the other hand, we have also

$$
\sigma_{2}\left(\tilde{g}^{-1} A_{\tilde{g}}\right)=\frac{1}{96} R_{\tilde{g}}^{2}-\frac{1}{8}\left|E_{\tilde{g}}\right|^{2} .
$$

Thus, we arrive to

$$
\kappa_{\left(P^{4}, P^{3}\right)}=\frac{1}{4} \int_{M}\left(\frac{1}{12} R_{\tilde{g}}^{2}-\left|E_{\tilde{g}}\right|^{2} \mathrm{~d} V_{\tilde{g}}\right) \leq \frac{1}{48} R_{\tilde{g}}^{2} \operatorname{Vol}_{\tilde{g}}(M) \leq \frac{192}{48} \pi^{2}=4 \pi^{2}
$$

and equality holds if and only if $(M, g)$ is conformally diffeomorphic to $\left(S_{+}^{4}, g_{S}\right)$. This completes the proof of the lemma.

Now coming back to our proof, we have that, since $Y(M, \partial M,[g])>0$, and $(M, g)$ has an umbilic boundary, then by Lemma $5.2 \kappa_{\left(P^{4}, P^{3}\right)} \leq 4 \pi^{2}$ and equality holds if and only if $(M, g)$ is conformally diffeomorphic to $S_{+}^{4}$ with its standard metric. Hence, we can assume that $\kappa_{\left(P^{4}, P^{3}\right)}<4 \pi^{2}$, otherwise there is noting to do. Thus taking $\alpha_{1}$ close to $16 \pi^{2}$ and $\alpha_{2}$ close to $12 \pi^{2}$, and using Cauchy inequality, trace theorem, Sobolev embedding, Poincaré inequality, and Lemma 2.9, we get

$$
I I(u) \geq \gamma_{0}\|u-\bar{u}\|_{H^{2}}-C_{0}
$$

for some $\gamma_{0}>0$ and some large $C_{0}$. Hence in any case we obtain

$$
I I(u) \geq \gamma_{\mid} \mid u-\bar{u} \|_{H^{2}}-C_{1}
$$

for some $\gamma_{1}>0$ and some large $C_{1}$. From this, and the fact that $I I$ is invariant by translation by constant, we have the existence of a minimizer $u_{n}$ such that

$$
\int_{M} e^{4 u_{n}} \mathrm{~d} V_{g}=1
$$

Thus by the coercivity property (5.4), we have

$$
\left\|u_{n}-\bar{u}_{n}\right\|_{H^{2}} \leq C \text {. }
$$

On the other hand, using Proposition 2.7, we infer

$$
\log \int_{M} e^{4\left(u_{n}-\bar{u}_{n}\right)} \mathrm{d} V_{g} \leq C .
$$

So using (5.5), (5.6) and Jensen's inequality we infer

$$
\left|\bar{u}_{n}\right| \leq C \text {. }
$$

Thus, we arrive to

$$
\left\|u_{n}\right\|_{H^{2}} \leq C .
$$


Hence up to a subsequence, we have

$$
u_{n} \rightarrow u \text { in } H^{2}
$$

Furthermore, we have $u \in H_{\frac{\partial}{\partial n}}$. On the other hand, it is easy to see that $I I$ is weakly lower semicontinuous on $H^{2}$. Thus we have $u$ is a minimizer of $I I$. This completes the proof of Corollary 1.10 .

\section{References}

[1] A. L. Besse, "Einstein Manifolds”, Springer Verlag, Berlin, 1987.

[2] T. P. BRAnson, "The Functional Determinant", Global Analysis Research Center, Lecture Notes Series, Vol. 4, Seoul National University, 1993.

[3] T. P. BRANSON, Differential operators canonically associated to a conformal structure, Math. Scand. 57 (1985), 293-345.

[4] T. P. BRANSON and B. OERSTED, Explicit functional determinants in four dimensions, Proc. Amer. Math. Soc. 113 (1991), 669-682.

[5] M. Berger, Les variétés riemanniennes $\frac{1}{4}$-pincées. Ann. Scuola Norm. Sup. Pisa 14 (1960), 161-170.

[6] S. BRENDLE and R. M. SCHOEN, Classification of manifolds with weakly $\frac{1}{4}$-pinched curvatures, Acta Math. 200 (2008), 1-13.

[7] C. BOHM and B. WILKING, Manifolds with positive curvature operator are space-forms, Ann. of Math. 167 (2008) 1079-1097.

[8] L. Caffarelli, L. Nirenberg and J. Spruck, The Dirichlet problem for nonlinear second order elliptic equations. III. Functions of the eigenvalues of the Hessian, Acta Math. 155 (1985), 261-301.

[9] G. CATino and Z. DJADl, Conformal deformations of integral pinched 3-manifolds, Adv. Math. 223 (2010), 393-404.

[10] G. Catino, Z. Djadli and C. B. NDiAye, A sphere theorem on locally conformally flat even-dimensional manifolds, 2008, preprint.

[11] S. S. Chen, Local estimates for some fully nonlinear elliptic equations, Int. Math. Res. Not. 55 (2005), 3403-3425.

[12] S. S. Chen, Conformal deformation on manifolds with boundary, Geom. Funct. Anal. 19 (2009), 1024-1064.

[13] S. Y. A. CHANG and J. QING, The zeta functional determinants on manifolds with boundary. I. The formula, J. Funct. Anal. 147 (1997), 327-362.

[14] S. Y. A. CHANG and J. QING, The zeta functional determinants on manifolds with boundary. II. Extremal metrics and compactness of isospectral set, J. Funct. Anal. 147 (1997), 363-399.

[15] S. Y. A. ChANG, M. J. GURSKY and P. C. YANG, A conformally invariant sphere theorem in four dimensions, Publ. Math. Inst. Hautes Études Sci. 98 (2003), 105-143.

[16] S. Y. A. ChANG and P. C. YANG, On a fourth order curvature invariant, In: "Comtemporary Mathematics", Vol. 237, Spectral Problems in Geometry and Arithmetic, Ed. T. Branson, AMS, 1999, 9-28.

[17] Z. DJADLI and A. MALCHIODI, Existence of conformal metrics with constant $Q$-curvature, Ann. of Math. 168 (2008), 813-858.

[18] J. F. EscoBAR, The Yamabe problem on manifolds with boundary, J. Differential Geom. 35 (1992), 21-84. 
[19] L. C. Evans, Classical solutions of fully nonlinear, convex, second-order elliptic equations. Comm. Pure Appl. Math. 35 (1982), 333-363.

[20] C. FefFerman and C. R. GRAhaM, Q-curvature and Poincaré metrics, Math. Res. Lett. 9 (2002), 139-151.

[21] C. FefFerman and C. Graham, Conformal invariants, In: "Élie Cartan et les mathématiques d'aujourd'hui", Asterisque, 1985, 95-116.

[22] R. S. Hamilton, Three manifolds with positive Ricci curvature, J. Differential Geom. 17 (1982), 255-306.

[23] Y. Ge, C.-S. LIN and G. WANG, On the $\sigma_{2}$-scalar curvature, J. Differential Geom. 84 (2010), 45-86.

[24] D. Gilbar and N. Trudinger, "Elliptic Partial Differential Equations of Second Order", $2^{\text {nd }}$ edition, Springer-Verlag, 1983.

[25] C. R. Graham, R. Jenne, L. Mason and G. Sparling, Conformally invariant powers of the Laplacian, I: existence, J. London Math. Soc. 46 (1992), 557-565.

[26] M. J. GURSKY and J.A. VIACLOVSKY, A fully nonlinear equation on four-manifolds with positive scalar curvature, J. Differential Geom. 63 (2003), 131-154.

[27] W. KLINGEnberg, Über Riemannsche Mannigfaltigkeiten mit nach oben beschränkter Krümmung. Ann. Mat. Pura Appl. 60 (1962), 49-59.

[28] N.V. KRYLOV, Boundedly inhomogeneous elliptic and parabolic equations in a domain, Izv. Akad. Nauk SSSR Ser. Mat. 47 (1983), 75-108.

[29] P. L. LIONS and N. S. TRUDINGER, Linear oblique derivative problems for the uniformly elliptic Hamilton-Jacobi-Bellman equation, Math. Z. 191 (1986), 11-15.

[30] C. MARGERIN, A sharp characterization of the smooth 4-sphere in curvature terms, Comm. Anal. Geom. 6 (1998), 21-65.

[31] C. B. NDIAYE, Conformal metrics with constant $Q$-curvature for manifolds with boundary, Comm. Anal. Geom. 16 (2008), 1049-1124.

[32] C. B. NDIAYE, Constant T-curvature conformal metric on 4-manifolds with boundary, Pacific J. Math. 240 (2009), 151-184.

[33] S. PANEITZ, A quartic conformally covariant differential operator for arbitrary pseudoRiemannian manifolds, SIGMA Symmetry Integrability Geom. Methods Appl. 4 (2008), 3 pages.

[34] S. PANEITZ, Essential unitarization of symplectics and applications to field quantization, J. Funct. Anal. 48 (1982), 310-359.

[35] H. E. RAUCH, A contribution to differential geometry in the large, Ann. of Math. 54 (1951), $38-55$.

[36] J. Viaclovsky, Conformal geometry, contact geometry, and the calculus of variations, Duke Math. J. 101 (2000), 283-316.

[37] Y. SHEN, On Ricci deformation of a Riemannian metric on manifold with boundary, Pacific J. Math. 173 (1996), 203-221.

[38] Y. TASHIRO, Complete Riemannian manifolds and some vector fields, Trans. Amer. Math. Soc. 117 (1965), 251-275.

SISSA

International School for Advanced Studies

Via Beirut, 2-4

34014 Trieste, Italia

catino@sissa.it

Mathematisches Institut der Universität Tübingen

Auf der Morgenstelle, 10

72076 Tübingen, Germany

ndiaye@everest.mathematik.uni-tuebingen.de 